Pacific Journal of Mathematics

GENERALIZED RIGID ELEMENTS IN FIELDS 


\title{
GENERALIZED RIGID ELEMENTS IN FIELDS
}

\section{KAZIMIERZ SZYMICZEK}

\begin{abstract}
Rigid elements in a field, should they exist, have strong influence on structure of the Witt ring of the field. We generalize rigid elements to the context of $n$-fold Pfister forms in two ways and study the relations between $n$-rigid and super $n$-rigid elements in various classes of fields including global fields, and in abstract Witt rings. In special cases the existence of higher rigidities turns out to be equivalent to important properties of fields known in literature. Among these are the property $A_{n}$, torsion freeness of $I^{n} F$ and finite stability index of $W F$.
\end{abstract}

Introduction. Rigid elements have proved to be of importance in studying the structure of the Witt ring of a field (see, for instance, [1], [2], [3], [4], [18]). However, the notion of rigidity has strictly binary character and almost all existing approaches to rigid elements revolve around the defining condition $D_{F}\langle 1, x\rangle=\dot{F}^{2} \cup x \dot{F}^{2}$. It is easy to find equivalent conditions which suggest the general notion of rigidity defined in terms of $n$-fold Pfister forms and powers of the fundamental ideal of Witt ring. Thus an element $x \in \dot{F}$ is said to be $n$-rigid if every $n$-fold Pfister form $\phi$ annihilated by $\langle 1, x\rangle$ in $W F$ has to have the factor $\langle 1,-x\rangle$ making $\phi \cdot\langle 1, x\rangle=0$ a triviality. Super $n$-rigidity is obtained by replacing Pfister forms with elements of $I^{n} F$. It turns out that what is easily seen to be equivalent for $n=1$ is presumably a hard problem when $n>2$. In this paper we make an attempt to understand what lies behind these general conditions.

In the first section we work in arbitrary fields (of characteristic not two). We find a characterization of $n$-rigid elements by value sets of quadratic forms and show that the sets of $n$-rigid elements form an ascending chain for $n=1,2, \ldots$. The same is proved for super $n$-rigid elements for special classes of fields. This leads to a new characterization of linked fields (Proposition 1.23). In special cases we arrive at interesting properties of fields studied in the literature. Thus $n$-rigidity of $x=1$ means the field satisfies Elman and Lam's property $A_{n}$ (cf. [8]) and super $n$-rigidity of $x=1$ means $I^{n} F$ is torsion free. And for $x=-1$ the two rigidity properties coincide with $W F$ being $(n-1)$-stable (cf. [6]). As a by-product we obtain a characterization of stability in terms of value groups of Pfister forms (see (1.11) below). 
Section 2 proves that $n$-rigid and super $n$-rigid elements coincide for every $n$, if the field in question is amenable (cf. [9]) or linked (cf. [11]). We also compute explicitly the sets of $n$-rigid elements for finite, $\mathfrak{p}$-adic and global fields.

In $\$ 3$ we study higher rigidities in abstract Witt rings in the sense of Marshall [15]. The main result asserts that $n$-rigid and super $n$-rigid elements coincide in every Witt ring of elementary type. This result includes, in particular, all Pythagorean fields with finite group of square classes and all fields with group of square classes of order $\leq 32$. We conclude this paper with relating our generalization of rigid elements to Arason, Elman and Jacob's generalization of birigid elements ([1]).

We use standard notation and terminology (except for higher rigidities). The fields considered are always assumed to have characteristic different from two and $\dot{F}=F \backslash\{0\} . P_{n} F$ denotes the set of all $n$-fold Pfister forms over $F$ and we often think of $P_{n} F$ as a subset of the Witt ring $W F$. For $\phi \in P_{n} F$, we write $\phi^{\prime}$ for the pure subform of $\phi$, thus $\phi \cong\langle 1\rangle \perp \phi^{\prime}$. The $n$th radical $R_{n} F$ is the intersection of value groups of all $n$-fold Pfister forms over $F$. Annihilator ideals $\mathrm{Ann}_{W F}\langle 1, x\rangle$ are wirtten simply Ann $\langle 1, x\rangle$. Unexplained notation and terminology follows [13], [14] and [15].

1. Rigidity and super rigidity. We begin with recalling conditions characterizing rigid elements in fields.

Proposition 1.1. For an arbitrary $x \in \dot{F}$, the following statements are equivalent.

(i) $D_{F}\langle 1, x\rangle=\dot{F}^{2} \cup x \dot{F}^{2}$, i.e., $x$ is rigid in $F$.

(ii) $0 \neq \phi \in P_{1} F$ and $-x \in D_{F} \phi \Rightarrow-x \in D_{F} \phi^{\prime}$.

(iii) $P_{1} F \cap \operatorname{Ann}\langle 1, x\rangle=\langle 1,-x\rangle P_{0} F$.

(iv) $I F \cap \operatorname{Ann}\langle 1, x\rangle=\langle 1,-x\rangle I^{0} F$.

Proof. Here $P_{0} F=\{0,\langle 1\rangle\}$ and $I^{0} F=W F$. (i) $\Rightarrow$ (ii) $\Rightarrow$ (iii) $\Rightarrow$ (i) and (iv) $\Rightarrow$ (iii) are all easily verified. For $\langle 1, x\rangle$ anisotropic, (i) $\Rightarrow$ (iv) is an immediate consequence of Witt Annihilator Theorem [14, p. 71]. For $x \in-\dot{F}^{2}$, (i) implies $F$ has at most two square classes and then $I F=2$. $W F$ which is (iv).

Conditions (ii), (iii) and (iv) are easily generalized to the context of $n$-fold Pfister forms. We will also generalize (i) but that requires introducing an appropriate analogue of the $n$th radical of a field. 
Definition 1.2. For $x \in \dot{F}$ and any non-negative integer $n, R_{0}(F, x)$ $=\dot{F}^{2} \cup x \dot{F}^{2}$, and

$$
R_{n}(F, x)=\bigcap\left\{D_{F} \phi \cdot D_{F}\left(\langle x\rangle \perp \phi^{\prime}\right): \phi \in P_{n} F\right\}, \quad \text { for } n \geq 1 .
$$

$R_{n}(F, x)$ is said to be the $n$th radical of $F$ centered at $x$.

Observe that for $x=1, R_{n}(F, 1)=R_{n} F$, is the $n$th radical of $F$. R. Bos [3, p. 65] proved that for every $\phi \in P_{n} F$ the set $D_{F} \phi \cdot D_{F}\left(\langle x\rangle \perp \phi^{\prime}\right)$ is a subgroup of $\dot{F}$ containing $D_{F} \phi$. Hence $R_{n}(F, x)$ is a subgroup of $\dot{F}$ containing $R_{n} F$. Clearly $x \in R_{n}(F, x)$ hence also

$$
R_{n} F \cup x R_{n} F \subseteq R_{n}(F, x) \text {. }
$$

Now we consider the four conditions generalizing (i) through (iv) in Proposition 1.1. For $x \in \dot{F}$ and $n \geq 1$ these are

(A) $D_{F}\langle 1, x\rangle \subseteq R_{n-1}(F, x)$.

(B) $0 \neq \phi \in P_{n} F$ and $-x \in D_{F} \phi \Rightarrow-x \in D_{F} \phi^{\prime}$.

(C) $P_{n} F \cap$ Ann $\langle 1, x\rangle \subseteq\langle 1,-x\rangle P_{n-1} F$.

(D) $I^{n} F \cap$ Ann $\langle 1, x\rangle \subseteq\langle 1,-x\rangle I^{n-1} F$.

Observe that for $n>1$ the assumption $\phi \neq 0$ in (B) is superfluous (but it is necessary when $n=1$ ). Also in (C) and (D) the right-hand sides are obviously contained in left-hand sides so that in (C) and (D) we can put the equality sign as well.

THEOREM 1.3. (A) $\Leftrightarrow(\mathrm{B}) \Leftrightarrow(\mathrm{C}) \Leftarrow(\mathrm{D})$.

Proof. We may assume $n>1$. For $\phi \in P_{n} F$ we have $\phi \cdot\langle 1, x\rangle=0$ $\Leftrightarrow-x \in D_{F} \phi$ and $-x \in D_{F} \phi^{\prime} \Leftrightarrow \phi \in\langle 1,-x\rangle P_{n-1} F$ (Pure Subform Theorem [14, p. 64]). This verifies (B) $\Leftrightarrow(\mathrm{C})$. To prove (D) $\Rightarrow(\mathrm{C})$, let $\phi \in P_{n} F$ $\cap \operatorname{Ann}\langle 1, x\rangle$. Then by (D), $\phi \in\langle 1,-x\rangle I^{n-1} F$, hence by [6, Theorem 2.1], $\phi \in\langle 1,-x\rangle P_{n-1} F$ as needed. Two lemmas will be needed to finish the proof of 1.3 .

LeMMA 1.4. Let $\phi \in P_{n} F$ and $n \geq 1$. Then for $x, y \in \dot{F}$,

$$
-x \in D_{F}\left(\phi^{\prime} \perp-y \phi\right) \Leftrightarrow y \in D_{F} \phi \cdot D_{F}\left(\langle x\rangle \perp \phi^{\prime}\right) \text {. }
$$

Proof. If $-x \in D_{F}\left(\phi^{\prime} \perp-y \phi\right)$, then $-x \in D_{F}\langle a,-y b\rangle$ for some $a \in$ $D_{F} \phi^{\prime}$ and $b \in D_{F} \phi$. Hence $y b \in D_{F}\langle x, a\rangle$ and $y \in b D_{F}\langle x, a\rangle \subseteq D_{F} \phi$. $D_{F}\left(\langle x\rangle \perp \phi^{\prime}\right)$. Conversely, if $y \in D_{F} \phi \cdot D_{F}\left(\langle x\rangle \perp \phi^{\prime}\right)$, then by $\in$ $D_{F}\left(\langle x\rangle \perp \phi^{\prime}\right)$ for some $b \in D_{F} \phi$ and so $-x \in D_{F}\left(\phi^{\prime} \perp\langle-b y\rangle\right) \subseteq$ $D_{F}\left(\phi^{\prime} \perp-y \phi\right)$.

LEMMA 1.5. Let $\phi \in P_{n} F, n \geq 1$ and $\langle 1, x\rangle \phi=0$. Then $\phi=\langle 1,-y\rangle \theta$ for some $y \in D_{F}\langle 1, x\rangle$ and $\theta \in P_{n-1} F$. 
Proof. This is well known. If $x \notin-\dot{F}^{2}$, we have $-x=t^{2}-y$ with $-y \in D_{F} \phi^{\prime}$ and so $\phi \in\langle 1,-y\rangle P_{n-1} F$ by Pure Subform Theorem. Also $y=t^{2}+x \in D_{F}\langle 1, x\rangle$. If $x \in-\dot{F}^{2}$, the result is trivially true.

Now we prove $(\mathrm{C}) \Rightarrow(\mathrm{A})$. Let $y \in D_{F}\langle 1, x\rangle$. Then for every $\phi \in$ $P_{n-1} F, \phi\langle 1,-y\rangle\langle 1, x\rangle=0$, i.e., $\phi\langle 1,-y\rangle \in P_{n} F \cap \operatorname{Ann}\langle 1, x\rangle$. Using (C), there exists $\theta \in P_{n-1} F$ such that $\phi\langle 1,-y\rangle=\langle 1,-x\rangle \theta$. It follows

$$
\phi^{\prime} \perp-y \phi=\theta^{\prime} \perp-x \theta,
$$

hence $-x \in D_{F}\left(\phi^{\prime} \perp-y \phi\right)$. By Lemma 1.4, $y \in D_{F} \phi \cdot D_{F}\left(\langle x\rangle \perp \phi^{\prime}\right)$. Since this holds for every $\phi \in P_{n-1} F$, we get $y \in R_{n-1}(F, x)$.

$(\mathrm{A}) \Rightarrow(\mathrm{C})$. Let $\phi \in P_{n} F \cap \operatorname{Ann}\langle 1, x\rangle$. By Lemma $1.5, \phi=\langle 1,-y\rangle \theta$ with $y \in D_{F}\langle 1, x\rangle$ and $\theta \in P_{n-1} F$. Using now (A), we get $y \in D_{F} \theta$. $D_{F}\left(\langle x\rangle \perp \theta^{\prime}\right)$ and so, by Lemma 1.4, $-x \in D_{F}\left(\theta^{\prime} \perp-y \theta\right)=D_{F} \phi^{\prime}$. Hence $\phi \in\langle 1,-x\rangle P_{n-1} F$. This finishes proof of Theorem 1.3.

Definition 1.6. We say $x \in \dot{F}$ is $n$-rigid if it satisfies one (hence all) of the conditions (A), (B), (C). The set of all $n$-rigid elements in $F$ will be written $R^{n} F$. We say $x \in \dot{F}$ is super $n$-rigid if it satisfies (D). The set of all super $n$-rigid elements is denoted $\operatorname{Sup} R^{n} F$.

Remarks (1.7). $R^{1} F=\operatorname{Sup} R^{1} F$ is the set of usual rigid elements in $F$ satisfying conditions of Proposition 1.1.

(1.8) For every $n \geq 1$, Sup $R^{n} F \subseteq R^{n} F$, according to (D) $\Rightarrow$ (C). A large portion of this paper is devoted to studying the cases where the converse inclusion holds.

(1.9) $n$-rigidity of $x=1$ is equivalent to

$$
P_{n} F \cap \operatorname{Ann}\langle 1,1\rangle=0 \text {. }
$$

This is precisely the definition of property $A_{n}$ studied by Elman and Lam in [8]. The equivalence of (A) and (C) includes as a special case the following result (cf. [8, Theorem 4.3]): $F$ satisfies $A_{n}$ iff $D_{F}\langle 1,1\rangle \subseteq R_{n-1} F$.

(1.10) Super $n$-rigidity of $x=1$ means $I^{n} F \cap A n n\langle 1,1\rangle=0$. This is easily seen to be equivalent with $I^{n} F \cap W_{t} F=0$. Thus the question of whether $1 \in R^{n} F \Rightarrow 1 \in \operatorname{Sup} R^{n} F$ turns out to be equivalent to the important open problem: does $A_{n}$ imply that $I^{n} F$ is torsion free? Elman and Lam [8, p. 37] say that this "seems to be a rather difficult and elusive question". Certainly, the same applies to the general question raised by Theorem 1.3: does (C) imply (D)? or equivalently, for any field $F$, is $R^{n} F \subseteq \operatorname{Sup} R^{n} F$ ? 
(1.11) For $x=-1$ we also arrive at an interesting and important point in quadratic form theory. Indeed, $-1 \in R^{n} F \Leftrightarrow P_{n} F=2 P_{n-1} F \Leftrightarrow I^{n} F=$ $2 I^{n-1} F \Leftrightarrow-1 \in \operatorname{Sup} R^{n} F$. Thus, for $x=-1$, (C) and (D) are equivalent and reduce to the condition: "WF is $(n-1)$-stable" (cf. [6, Def,. 3.8]). In terms of value groups of Pfister forms we get the following result (using (A) with $x=-1)$ : $W F$ is $(n-1)$-stable iff for every $(n-1)$-fold Pfister form $\phi$,

$$
D_{F} \phi D_{F}\left(\langle-1\rangle \perp \phi^{\prime}\right)=\dot{F}
$$

In case $n=2$, the condition says

$$
D_{F}\langle 1, a\rangle \cdot D_{F}\langle 1,-a\rangle=\dot{F}, \quad \text { for every } a \in \dot{F} .
$$

This complements the list of equivalent statements for 1-stability of WF given in [6, Prop. 3.9].

(1.12) If $F$ satisfies $A_{n}$ (i.e., $1 \in R^{n} F$ ), then $\sum \dot{F}^{2} \subseteq R^{n} F$. Indeed, if $x \in \sum \dot{F}^{2}$ and $F$ satisfies $A_{n}$, then by [8, Theorem 4.3], $F$ satisfies $A_{n}(x)$, i.e., $\operatorname{Ann}\langle 1, x\rangle \cap P_{n} F=0$. Hence $x$ satisfies (C) and so $x \in R^{n} F$.

(1.13) If $F$ is non-real and satisfies $A_{n}$, then $R^{n} F=\dot{F}$. This follows immediately from (1.12).

(1.14) $R^{n} F$ and $\operatorname{Sup} R^{n} F$ are unions of cosets of $R_{n-1} F$ in $\dot{F}$. For, if $q \in I^{n-1} F$ and $y \in R_{n-1} F$, then $y q=q$ and so for $q \in I^{n} F$, $q \in \operatorname{Ann}\langle 1, x\rangle \Leftrightarrow q \in \operatorname{Ann}\langle 1, x y\rangle$ and $q \in\langle 1,-x\rangle I^{n-1} F \Leftrightarrow q \in$ $\langle 1,-x y\rangle I^{n-1} F$. Hence if $x$ satisfies (D), so does $x y$. The same applies to $R^{n} F$.

(1.15) If $1 \in R^{n} F\left(1 \in \operatorname{Sup} R^{n} F\right)$, then $R_{n-1} F \subseteq R^{n} F\left(R_{n-1} F \subseteq\right.$ Sup $\left.R^{n} F\right)$. This follows from (1.14).

Proposition 1.16. For every $n \geq 1, R^{n} F \subseteq R^{n+1} F$. In particular, $R^{1} F \subseteq R^{n} F$, for every $n \geq 1$.

Proof. Assume $x \in R^{n} F$ and $\phi \in P_{n+1} F \cap \operatorname{Ann}\langle 1, x\rangle$. Lemma 1.5 yields $\phi=\left\langle\left\langle-y, y_{2}, \ldots, y_{n+1}\right\rangle\right\rangle$ with $y \in D_{F}\langle 1, x\rangle$ and some $y_{i} \in \dot{F}$. Then $\theta:=\left\langle\left\langle-y, y_{2}, \ldots, y_{n}\right\rangle\right\rangle \in P_{n} F \cap \operatorname{Ann}\langle 1, x\rangle$ and since $x \in R^{n} F$ it follows that $\theta \in\langle 1,-x\rangle P_{n-1} F$. Thus $\phi=\theta\left\langle\left\langle y_{n+1}\right\rangle\right\rangle \in\langle 1,-x\rangle P_{n} F$, i.e., $x \in R^{n+1} F$. 
REMARK (1.17). A special case of 1.16 says $1 \in R^{n} F$ implies $1 \in$ $R^{n+1} F$, i.e., if $F$ satisfies $A_{n}$, it also satisfies $A_{m}$ for all $m \geq n$ (cf. [5, Lemma 2.10]). Combining (1.14) and Proposition 1.16 we get for every $n \geq 2$,

$$
R_{n-1} F \cdot R^{n-1} F \subseteq R^{n} F .
$$

It is to be remarked that the analogue of Proposition 1.16 for super rigid elements is not known to hold. What can be proved for an arbitrary field $F$ is summarized below.

Proposition 1.18. For any field $F$,

(i) $\operatorname{Sup} R^{1} F=R^{1} F$.

(ii) $\operatorname{Sup} R^{2} F=R^{2} F$.

(iii) $\operatorname{Sup} R^{1} F \subseteq \operatorname{Sup} R^{2} F \subseteq \operatorname{Sup} R^{3} F$.

Proof. (i) has already been noticed in (1.7). (ii) We want $R^{2} F \subseteq$ Sup $R^{2} F$. So let $x \in R^{2} F$ and assume first that $x \notin-\dot{F}^{2}$. Then $\operatorname{Ann}\langle 1, x\rangle$ is a 1-Pfister ideal (cf. [11, 2.4(a)]) and so by [11, Corollary 2.15], we get

$$
I^{2} F \cap \operatorname{Ann}\langle 1, x\rangle=\operatorname{Ann}\langle 1, x\rangle \cdot I F .
$$

That this implies $x \in \operatorname{Sup} R^{2} F$ is shown in the proof of Theorem 2.2 in the next section. Now, if $x \in-\dot{F}^{2}$, then for every $n \geq 1, x \in R^{n} F \Rightarrow x \in$ Sup $R^{n} F$ according to (1.11). This proves (ii). (iii) With (i), (ii) and Proposition 1.16 , we only need to show that $\operatorname{Sup} R^{2} F \subseteq \operatorname{Sup} R^{3} F$. This follows from Theorem 1.22 and Example 1.21 below.

For the balance of this section, we concentrate on conditions under which $\operatorname{Sup} R^{n} F \subseteq \operatorname{Sup} R^{n+1} F$ holds.

Definition 1.19. An ideal $A$ in $W F$ is said to be $n$-Pfister neighbor ideal, if for every anisotropic $q \in A$ with $\operatorname{dim} q>2^{n-1}$, there is an isometry

$$
q \cong a \phi \perp \theta
$$

where $a \in \dot{F}, \phi$ is a Pfister neighbor associated to an $n$-fold Pfister form and $\theta$ is an arbitrary form.

EXAMPLES. (1.20). Every strong $n$-Pfister ideal is (trivially) $n$-Pfister neighbor ideal. More interestingly, every strong $n$-Pfister ideal $A$ is $(n+1)$-Pfister neighbor ideal. Indeed, let $q \in A, \operatorname{dim} q>2^{n}$ and $q=$ $a_{1} \phi_{1} \perp \cdots \perp a_{r} \phi_{r}$, where $a_{i} \in \dot{F}, \phi_{i} \in P_{n} F$ and $r>1$. Consider $a_{1} \phi_{1} \perp$ $a_{2} \phi_{2}=a_{1}\left(\phi_{1} \perp a_{1} a_{2} \phi_{2}\right)$ and put $\phi=\phi_{1} \perp\left\langle a_{1} a_{2}\right\rangle$. Then $\phi$ is a Pfister 
neighbor associated to $\phi_{1} \cdot\left\langle 1, a_{1} a_{2}\right\rangle$ and (1.19.1) holds with obvious choices for $\boldsymbol{a}$ and $\boldsymbol{\theta}$.

(1.21). IF is 2-Pfister neighbor ideal, since $I F$ is strong 1-Pfister. For the cases where $I^{2} F$ is 3-Pfister neighbor ideal, see Proposition 1.23 below.

THEOREM 1.22. If $I^{n-1} F$ is n-Pfister neighbor ideal, then

$$
\operatorname{Sup} R^{n} F \subseteq \operatorname{Sup} R^{n+1} F \text {. }
$$

Proof. Let $x \in \operatorname{Sup} R^{n} F$ and $q \in I^{n+1} F \cap \operatorname{Ann}\langle 1, x\rangle$. Then $q \in I^{n} F$ $\cap \operatorname{Ann}\langle 1, x\rangle=\langle 1,-x\rangle I^{n-1} F$, and so $q=\langle 1,-x\rangle p$, where $p$ is anisotropic form in $I^{n-1} F$. We prove $q \in\langle 1,-x\rangle I^{n} F$ by induction on $\operatorname{dim} p$. If $\operatorname{dim} p \leq 2^{n-1}$, then $\operatorname{dim} q \leq 2^{n}$ and since $q \in I^{n+1} F$, we conclude $q=0$ (by Arason-Pfister Haupsatz) and we are done. So assume $\operatorname{dim} p>$ $2^{n-1}$. Then by the hypothesis on $I^{n-1} F, p \cong a \phi \perp \theta$, where $\phi$ is a Pfister neighbor of some $\sigma \in P_{n} F, a \in \dot{F}$ and $\theta$ is an $F$-form. Thus $\sigma \cong \phi \perp \phi_{1}$ with $\operatorname{dim} \phi_{1}<\operatorname{dim} \phi$ and for $p_{1}=p \perp-a \sigma$ we have $p_{1}=-a \phi_{1}+\theta$ in $W F$ and

$$
\operatorname{dim}\left(p_{1}\right)_{a n} \leq \operatorname{dim} \phi_{1}+\operatorname{dim} \theta<\operatorname{dim} \phi+\operatorname{dim} \theta=\operatorname{dim} p .
$$

Now

$$
\begin{aligned}
\langle 1,-x\rangle p_{1} & =\langle 1,-x\rangle p-a\langle 1,-x\rangle \sigma \\
& =q-a\langle 1,-x\rangle \sigma \in I^{n+1} F \cap \operatorname{Ann}\langle 1, x\rangle
\end{aligned}
$$

and so, by induction, $\langle 1,-x\rangle p_{1} \in\langle 1,-x\rangle I^{n}$. Hence also $q=\langle 1,-x\rangle p_{1}$ $+a\langle 1,-x\rangle \sigma \in\langle 1,-x\rangle I^{n} F$, as required.

Recall that (1.21) and Theorem 1.22 imply our earlier statement in Proposition 1.18 (iii). The question now arises what other cases are covered by the Theorem. We answer this completely for $n=3$ proving the following result.

Proposition 1.23. $I^{2} F$ is a 3-Pfister neighbor ideal if and only if $I^{2} F$ is linked (iff quaternion algebras form a subgroup in the Brauer group of $F$ ).

Proof. If $I^{2} F$ is linked, then by [7, Theorem 2.7], $I^{2} F$ is strong 2-Pfister ideal in the sense of [11] and so by (1.20) it is 3-Pfister neighbor ideal.

To prove the converse, it is sufficient to show that if $\theta_{1}, \theta_{2} \in P_{2} F$, then the form $q=\theta_{1}^{\prime} \perp\langle-1\rangle \theta_{2}^{\prime}$ satisifes $q \equiv \phi \bmod I^{3} F$ for some $\phi \in P_{2} F$ (or even a 4-dimensional form in $I^{2} F$ ) by [17, Zusatz, p. 124]. If $q$ is 
anisotropic 6-dimensional, up to a scalar, it contains a 5-dimensional Pfister neighbor of a 3-fold Pfister form. Since $q$ lies in $I^{2} F$, going mod $I^{3} F$ yields the result.

COROLlaRY 1.24. If $I^{2} F$ is linked, then

$$
\operatorname{Sup} R^{3} F \subseteq \operatorname{Sup} R^{4} F \text {. }
$$

This is an immediate consequence of 1.22 and 1.23. However, we will recapture and strengthen this result in the next section (see Corollary 2.6 and combine it with Proposition 1.16).

2. Amenable and linked fields. In this section we prove that basic question of whether $R^{n} F=\operatorname{Sup} R^{n} F$ for every $n \geq 1$, has an affirmative answer for every amenable and every linked field. We also compute the sets $R^{n} F$ for all $\mathfrak{p}$-adic and all global fields.

Recall that a field $F$ is said to be amenable, if for every finite set $\left\{\phi_{1}, \ldots, \phi_{k}\right\}$ of Pfister forms over $F$ and for $K=F\left(\phi_{1}, \ldots, \phi_{k}\right)$, the iterated function field of $\phi_{1}, \ldots, \phi_{k}$ over $F$,

$$
W(K / F)=\sum \phi_{i} \cdot W F,
$$

where $W(K / F)$ is the kernel of the canonical ring homomorphism $W F \rightarrow$ WK (see [9] for details). We will use below corollary to a basic result of [9] on amenable fields.

LEMMA 2.1 ([9, Cor. 4.6]). If $F$ is amenable and $\phi$ is an anisotropic Pfister form over $F$, then for any integer $n \geq 1$,

$$
I^{n} F \cap \operatorname{Ann} \phi=\operatorname{Ann} \phi \cdot I^{n-1} F .
$$

Using this we prove the following result.

THEOREM 2.2. If $F$ is an amenable field, then for every integer $n \geq 1$, $R^{n} F=\operatorname{Sup} R^{n} F$.

Proof. We only need to show $R^{n} F \subseteq \operatorname{Sup} R^{n} F$. So suppose $x \in \dot{F}$ satisfies (C) of Theorem 1.3 and let $q \in I^{n} F \cap \operatorname{Ann}\langle 1, x\rangle$. If $x \notin-\dot{F}^{2}$, Lemma 2.1 applies, hence $q \in \operatorname{Ann}\langle 1, x\rangle \cdot I^{n-1} F$. Thus $q=\sum A B$ with $A \in \operatorname{Ann}\langle 1, x\rangle$ and $B \in I^{n-1} F$. By Witt Annihilator Theorem,

$$
A=\sum a_{i}\left\langle\left\langle-c_{i}\right\rangle\right\rangle, \quad \text { where } a_{i} \in \dot{F} \text { and } c_{i} \in D_{F}\langle 1, x\rangle \text {. }
$$

We also have

$$
B=\sum \pm \phi_{j}, \quad \text { where } \phi_{j} \in P_{n-1} F
$$


Since $\left\langle\left\langle-c_{l}\right\rangle\right\rangle \phi_{j} \in P_{n} F \cap \operatorname{Ann}\langle 1, x\rangle$ and $x$ is $n$-rigid, we get $\left\langle\left\langle-c_{l}\right\rangle\right\rangle \phi_{J} \in$ $\langle 1,-x\rangle P_{n-1} F$. It follows

$$
A B=\sum \pm a_{i}\left\langle\left\langle-c_{i}\right\rangle\right\rangle \phi_{j} \in\langle 1,-x\rangle I^{n-1} F,
$$

hence also $q \in\langle 1,-x\rangle I^{n-1} F$ as needed. If $x \in-\dot{F}^{2} \cap R^{n} F$, we get $x \in$ Sup $R^{n} F$ by (1.11).

COROLlaRY 2.3. The conclusion of Theorem 2.2 holds for

(i) all global fields (of characteristic not two),

(ii) all fields of transcendence degree $\leq 1$ over a real closed field,

(iii) all fields with the property that every anisotropic 4-dimensional form has determinant 1 (e.g., all $\mathfrak{p}$-adic fields).

Proof. The fields in (i) and (ii) are amenable by [10, Cor. 6.4] and those in (iii) by [9, Theorem A.14].

Now we proceed to linked fields. A field $F$ is said to be $n$-linked, if any pair of $n$-fold Pfister forms over $F$ are linked (cf. [7]).

THEOREM 2.5. Suppose $F$ is an $n$-linked field, $n \geq 2$. Then $R^{m} F=$ Sup $R^{m} F$ for every $m \geq n$.

Proof. Since for $n \geq 2, F$ being $n$-linked implies that $F$ is $(n+1)$ linked, it is sufficient to prove the Theorem in the case $m=n$. So assume $x \in R^{n} F$ and take an $q \in I^{n} F \cap \operatorname{Ann}\langle 1, x\rangle$. By [5, Lemma 4.4], there exists a representation

$$
q=a_{0} q_{0}+a_{1} q_{1}+\cdots+a_{r} q_{r}
$$

where $a_{l} \in \dot{F}$ and $q_{l} \in P_{n+i} F$. We induct on $r$. If $r=0, q_{0}=a_{0} q \in P_{n} F$ $\cap \operatorname{Ann}\langle 1, x\rangle$, hence $q_{0} \in\langle 1,-x\rangle P_{n-1} F$ by $n$-rigidity of $x$. Hence also $q \in\langle 1,-x\rangle I^{n-1} F$ as needed. Now assume $r>0$. Then from $q \in$ Ann $\langle 1, x\rangle$ we get the equation

$$
a_{0}\langle 1, x\rangle q_{0}+\cdots+a_{r-1}\langle 1, x\rangle q_{r-1}=-a_{r}\langle 1, x\rangle q_{r} \text { in } W F .
$$

By a dimension count it follows $\langle 1, x\rangle q_{r}$ is isotropic, hence hyperbolic. Thus $q_{r} \in P_{n+r} F \cap \operatorname{Ann}\langle 1, x\rangle=\langle 1,-x\rangle P_{n+r-1} F$, the latter by Proposition 1.16. Hence $a_{r} q_{r} \in\langle 1,-x\rangle I^{n-1} F$ and by induction, $q \in\langle 1,-x\rangle I^{n-1} F$ as needed.

Corollary 2.6. If $F$ is linked (i.e., $F$ is 2-linked or, equivalently, the quaternion algebras form a subgroup in the Brauer group of $F$ ), then for every $n \geq 1$,

$$
R^{n} F=\operatorname{Sup} R^{n} F
$$


For a list of examples of linked fields including finite, $\mathfrak{p}$-adic and global fields, see [7].

Now that we have settled that $n$-rigid and super $n$-rigid elements coincide in the fields of number theory, we proceed to computing $R^{n} F$ for $F$ finite, $\mathfrak{p}$-adic and global field.

Proposition 2.7. Let $F$ be a finite field (of characteristic not two). Then $R^{1} F=\dot{F} \backslash \dot{F}^{2}$ and $R^{n} F=\dot{F}$ for every $n \geq 2$.

Proof. The first statement follows by inspecting the value groups of (two) forms $\langle 1, x\rangle$. Since $F$ satisfies $A_{2}, R^{2} F=\dot{F}$ by (1.13), and the Proposition 1.16 gives the result for $n>2$.

Proposition 2.8. Let $F$ be a $\mathfrak{p}$-adic field (i.e., local with finite residue class field).

(i) If $F$ is non-dyadic, then

$$
R^{1} F= \begin{cases}\dot{F} \backslash \dot{F}^{2} & \text { if }-1 \in \dot{F}^{2} \\ \dot{F} \backslash \pm \dot{F}^{2} & \text { if }-1 \notin \dot{F}^{2}\end{cases}
$$

(ii) If $F$ is dyadic, then $R^{1} F=\varnothing$.

(iii) For any p-adic field $F, R^{2} F=\dot{F} \backslash \dot{F}^{2}$.

(iv) For any $\mathfrak{p}$-adic field $F$ and for any integer $n \geq 3, R^{n} F=\dot{F}$.

Proof. Assuming well-known facts about p-adic fields (cf. [13, Ch. VI]), we see that (i) and (ii) are routine. Since $I^{3} F=0$ and the unique anisotropic 2-fold Pfister form $\phi$ over $F$ satisfies $D_{F}\left(\phi^{\prime}\right)=\dot{F} \backslash \dot{F}^{2}$, we see that (iii) and (iv) also follows.

Proposition 2.9. Let $F$ be a global field (char $F \neq 2$ ). Then

(i) $R^{1} F=R^{2} F=\varnothing$.

(ii) $R^{n} F=\dot{F}$ for $n \geq 3$.

Proof. (i) Let $x \in \dot{F}$. By [16, 65:19] there exists a non-archimedean prime $\mathfrak{p}_{1}$ such that $x \in \dot{F}_{\mathfrak{p}_{1}}^{2}$. Let $\mathfrak{p}_{2}$ be another non-archimedean prime. By $[16,72: 1]$, there exists $\phi \in P_{2} F$ which is anisotropic over $F_{\mathfrak{p}}$ for $\mathfrak{p}=\mathfrak{p}_{1}$ or $\mathfrak{p}_{2}$ but hyperbolic over all other localizations $F_{\mathfrak{p}}$. In particular, $\langle 1, x\rangle \phi$ is torsion in torsion-free $I^{3} F$ hence hyperbolic. Now $-1 \notin D_{F_{p_{1}}}\left(\phi^{\prime}\right)$, so $\phi \notin\langle 1,-x\rangle I F$. This proves (i), since $R^{1} F \subseteq R^{2} F$. 
(ii) If $\phi \in P_{n} F, n \geq 3$, then $\phi=2 \gamma, \gamma \in P_{n-1} F$ (cf. 13, p. 172]). Suppose $\langle 1, x\rangle \phi=0$. Then $I^{3} F$ torsion-free implies that $\langle 1, x\rangle \gamma=0$. Thus Lemma 1.5 produces $y$ so that $\phi=2 \gamma=2\left\langle\left\langle-x-y^{2}\right\rangle\right\rangle \theta=$ $\left\langle\left\langle-x,-x-y^{2}\right\rangle\right\rangle \theta$, for some $\theta \in P_{n-2} F$ and (ii) is established.

REMARK (2.10). A part of the results in 2.7, 2.8 and 2.9 can be generalized to non-real $n$-linked fields. If $F$ is non-real $n$-linked, then by [7, Cor. 2.5], $I^{n+2} F=0$. It follows then that $R^{n+2} F=\operatorname{Sup} R^{n+2} F=\dot{F}$. In the case where $F$ is linked and non-real, we get

$$
R^{n} F=\dot{F} \quad \text { for every } n \geq 4 \text {. }
$$

3. Abstract Witt rings. We shall use the theory of abstract Witt rings as presented in [15]. For a Witt ring $W$ we write $G_{W}$ for the elementary 2-group of invertible elements in $W$ generating additively $W$ and $I W$ for the ideal of even dimensional elements in $W$. The set of $n$-fold Pfister elements, i.e., those of the form $\left(1+a_{1}\right) \cdots\left(1+a_{n}\right)$, where $a_{i} \in G_{W}$ is denoted $P_{n} W$. For $\phi=b_{1}+\cdots+b_{n}$ with $b_{i} \in G_{W}$ we will also use the form notation $\phi=\left\langle b, \ldots, b_{n}\right\rangle$ and call $\phi$ an $n$-dimensional form.

We say that $x \in G_{W}$ is $n$-rigid if

$$
P_{n} W \cap \operatorname{Ann}\langle 1, x\rangle=\langle 1,-x\rangle P_{n-1} W,
$$

and $x \in G_{W}$ is said to be super $n$-rigid, if

$$
I^{n} W \cap \operatorname{Ann}\langle 1, x\rangle=\langle 1,-x\rangle I^{n-1} W .
$$

The sets $R^{n} W$ and $\operatorname{Sup} R^{n} W$ are introduced similarly as in the field case. Let us notice that the (ABCD)-Theorem 1.3 carries over to the abstract case. Proof of 1.3 requires three essential tools, all available in the abstract situation: Pure Subform Theorem (cf. [15, p. 45] or [12, Prop. 4.13]), Witt Annihilator Theorem (cf. [15, p. 85] or [12, Theorem 2.15]) and Theorem 2.1 of [6] (cf. [12, Prop. 4.13]). In fact, all the results of $\$ 1$ through and including Proposition 1.18 (i) and (ii) carry over to abstract Witt rings. The remaining results require Arason-Pfister Hauptsatz (for $I^{n} F$ with $n \geq 3$ ) or function fields which are not available in the abstract case.

Let $\mathscr{W}$ be the category of Witt rings. This category has direct products $([15$, p. 99$])$ denoted by $\times$. If $H$ is a finite elementary 2-group, then the group ring $W[H]$ is also an abstract Witt ring. An abstract Witt ring is said to be of elementary type if it is in the smallest subcategory $\mathscr{E}$ of $\mathscr{W}$ containing rings isomorphic to Witt rings of finite fields, $\mathfrak{p}$-adic fields, the reals, the complexes and closed under the direct products and finite group ring formation. 
In this section we prove the following result.

THEOREM 3.1. For every abstract Witt ring $W$ of elementary type and every integer $n \geq 1$,

$$
R^{n} W=\operatorname{Sup} R^{n} W
$$

Recall that $W$ is said to be reduced if $\mathrm{Nil} W=0$. Since every finitely generated reduced Witt ring is of elementary type ([15, p. 165]) we get the following.

COROLLARY 3.2. Every finitely generated reduced Witt ring $W$ satifies (3.1.1) for every $n \geq 1$.

COROLlary 3.3. If $F$ is any pythagorean field with $\dot{F} / \dot{F}^{2}$ finite, then $R^{n} F=\operatorname{Sup} R^{n} F$ for every $n \geq 1$.

Proof. This follows from 3.2 since Witt rings of pythagorean fields are reduced.

Corollary 3.4. If $F$ is any field with $\left|\dot{F} / \dot{F}^{2}\right| \leq 32$, then for every $n \geq 1, R^{n} F=\operatorname{Sup} R^{n} F$.

Proof. From [4] and the earlier results cited there we know that every field $F$ with the group of square classes of order not greater than 32 determines Witt ring of elementary type. Hence 3.1 applies.

Corollary 3.5. For any field $F$ with $\left|\dot{F} / \dot{F}^{2}\right| \leq 32$ and every $n \geq 1$, if $F$ satisfies $A_{n}$, then $I^{n} F$ is torsion-free.

Indeed, by 3.4 , if $1 \in R^{n} F$, then $1 \in \operatorname{Sup} R^{n} F$.

To prove Theorem 3.1 we apply the usual method of handling elementary Witt rings. Thus we look at the behavior of rigid and super rigid elements under direct product and group ring operations and determine rigidities for basic indecomposable Witt rings. Putting together these will prove 3.1. The details are recorded in the following three propositions.

Proposition 3.6. Let $\left(W_{1}, G_{1}\right)$ and $\left(W_{2}, G_{2}\right)$ be abstract Witt rings and let $(W, G)=\left(W_{1} \times W_{2}, G_{1} \times G_{2}\right)$ be their product. Then for every $n \geq 1$ :

(i) $P_{n} W=P_{n} W_{1} \times P_{n} W_{2}$.

(ii) $I^{n} W=I^{n} W_{1} \times I^{n} W_{2}$. 
(iii) $R^{n} W=R^{n} W_{1} \times R^{n} W_{2}$ for $n \geq 2$.

(iv) $\operatorname{Sup} R^{n} W=\operatorname{Sup} R^{n} W_{1} \times \operatorname{Sup} R^{n} W_{2}$ for $n \geq 2$.

(v) If both $W_{1}$ and $W_{2}$ satisfy (3.1.1), then so does $W$.

Proposition 3.7. Let $(S, G)$ be an abstract Witt ring and $\Delta=\{1, t\}$ be a 2-element group. Let $W=S[\Delta]$ be the group ring. For $n>1$, if $R^{n-1} S=\operatorname{Sup} R^{n-1} S$ and $R^{n} S=\operatorname{Sup} R^{n} S$, then (3.1.1) holds for $W$.

PROPOSITION 3.8. For every $n \geq 1,(3.1 .1)$ is satisfied for every abstract Witt ring $W$ which is isomorphic to the Witt ring of a field from the following list: $\mathbf{R}, \mathbf{C}$, finite fields, $\mathfrak{p}$-adic fields.

Since the result 3.8 has been established in $§ 2$, we will prove only 3.6 and 3.7.

Proof of 3.6. (i) and (ii) are immediate consequences of the definition of direct product. (iii) and (iv) are routine on using (C) and (D) and the following result on annihilators. If $q=\left(q_{1}, q_{2}\right) \in W=W_{1} \times W_{2}$, then

$$
\mathrm{Ann}_{W} q=\mathrm{Ann}_{W_{1}} q_{1} \times \mathrm{Ann}_{W_{2}} q_{2} .
$$

Here one inclusion is trivial and to prove " $\supseteq$ " one uses the fact that Witt ring does not contain zero divisors of odd dimension. Now (v) follows from (iii) and (iv).

Now we proceed to the case of group rings. So let $\left(S, G_{S}\right)$ be a Witt ring and $\Delta=\{1, t\}$ be a 2-element group. Then $W=S[\Delta]$ is also a Witt ring and $G_{W}=G_{S} \times \Delta=G_{S} \cup t G_{S}$. In order to prove 3.7 we need the following lemma determining $n$-rigid elements in $W=S[\Delta]$.

LEMMA 3.9. For every $n>1, R^{n} W=R^{n-1} S \cup t G_{S}$.

Proof. Since $t G_{S} \subseteq R^{1} W \subseteq R^{n} W$, we need only to prove that

$$
R^{n} W \cap G_{S}=R^{n-1} S .
$$

So let $x \in R^{n-1} S$ and let $\phi \in P_{n} W \cap \operatorname{Ann}\langle 1, x\rangle$. If $\phi \in P_{n} S$, we get $\phi \in\langle 1,-x\rangle P_{n-1} S$, by 1.16 . If $\phi \notin P_{n} S$, we may write $\phi=\langle 1, y t\rangle \sigma$, $y \in G_{S}, \sigma \in P_{n-1} S$. Now $\langle 1, x\rangle \phi=0$ implies $\langle 1, x\rangle \sigma=0$ and it follows that

$$
\phi=\langle 1, y t\rangle_{\sigma} \in\langle 1, y t\rangle\langle 1,-x\rangle P_{n-2} S \subseteq\langle 1,-x\rangle P_{n-1} W .
$$

Conversely, if $x \in R^{n} W \cap G_{S}$ and $\phi \in P_{n-1} S \cap \operatorname{Ann}\langle 1, x\rangle$ then $\langle 1, t\rangle \phi$ $=\langle 1,-x\rangle \theta$ for some $\theta \in P_{n-1} W$ by hypothesis. If $\theta \in P_{n-1} S$, then $\phi=0$ and we are done. Otherwise $\langle 1,-x\rangle \theta=\langle\langle a,-x\rangle\rangle \sigma-a\langle\langle t,-x\rangle\rangle \sigma$ for some $a \in G_{S}$ and $\sigma \in P_{n-2} S$ and it follows $\phi=\langle 1,-x\rangle \sigma$, as required. 
Now we are ready to prove 3.7. So assume $n>1, R^{n-i} S=\operatorname{Sup} R^{n-i} S$, $i=0,1$ and $x \in R^{n} W$. Let $q \in I^{n} W \cap \operatorname{Ann}\langle 1, x\rangle$. By [15, p. 113],

$$
I^{n} W=I^{n} S \oplus\langle 1,-t\rangle I^{n-1} S,
$$

hence $q=q_{1}+\langle 1,-t\rangle q_{2}$, where $q_{1} \in I^{n} S$ and $q_{2} \in I^{n-1} S$. If $x \in G_{S}$, then $\langle 1, x\rangle q_{1}=0=\langle 1, x\rangle q_{2}$ and we finish by (3.9.1), since $R^{n-1} S \subseteq R^{n} S$. So assume $x=t y, y \in G_{S}$. Now $\langle\langle-t, x\rangle\rangle=\langle\langle-y, x\rangle\rangle$, so

$$
0=\langle 1, x\rangle q=\langle 1, x\rangle q_{1}+\langle\langle x,-y\rangle\rangle q_{2}=\langle 1, x\rangle\left(q_{1}+\langle\langle-y\rangle\rangle q_{2}\right) .
$$

But $q_{1}+\langle\langle-y\rangle\rangle q_{2} \in S$, so $q_{1}+\langle\langle-y\rangle\rangle q_{2}=0$ and $q=y\langle\langle-y t\rangle\rangle q_{2} \in$ $\langle\langle-x\rangle\rangle I^{n-1} S$. This finishes the proof of 3.7 .

Proof of Theorem 3.1. We induct on $n$. For $n=1$ the result holds for every abstract Witt ring $W$ (cf. (1.7)). So let $n>1$. We prove (3.1.1) by induction on $\left|G_{W}\right|$. If $\left|G_{W}\right| \leq 2$, then by Proposition 3.8 the result is true for every $n$. If $W$ is an elementary Witt ring and $\left|G_{W}\right|>2$, then either

(1) $W$ is basic indecomposable, or

(2) $W=W_{1} \times W_{2}$ with $\left|G_{W_{i}}\right|<\left|G_{W}\right|, i=1,2$, or

(3) $W=S[\Delta]$ with $|\Delta|=2$.

In the first case $W$ satisfies (3.1.1) by Proposition 3.8 (since $W$ is the Witt ring of a p-adic field). In the second case, by induction on $\left|G_{W}\right|, W_{1}$ and $W_{2}$ satisfy (3.1.1), hence by Proposition $3.6(\mathrm{v})$, so does $W$. In the third case, $\left|G_{S}\right|<\left|G_{W}\right|$, hence by induction on $\left|G_{W}\right|$ we have $R^{n} S=\operatorname{Sup} R^{n} S$ and by induction on $n, R^{n-1} S=\operatorname{Sup} R^{n-1} S$. Hence Proposition 3.7 applies and gives the result. This completes the proof of Theorem 3.1.

REMARKS. (3.10). We can make the result in 3.9 complete by observing that

$$
R^{1} W=R^{1} S \cup t G_{S} \text { provided }-1 \notin R^{1} S .
$$

This combined with 3.9 leads to an interesting corollary:

$$
R^{2} W=R^{1} W \quad \text { provided }-1 \notin R^{1} S .
$$

The excluded case $-1 \in R^{1} S$ can happen only when $\left|G_{S}\right| \leq 2$ and it is easy to find $R^{1} W$ by inspecting the value groups of binary forms over $S$.

(3.11) The results in 3.7, 3.9 and (3.10) apply to fields of formal power series $K=F((t))$. For example,

$$
\text { if }\left|\dot{F} / \dot{F}^{2}\right| \geq 4, \quad \text { then } R^{2} K=R^{1} K=R^{1} F \cup t \dot{F} \dot{K}^{2} .
$$


Finally, we discuss briefly the relations between $n$-rigid elements of an abstract Witt ring and the $n$-rigid elements in the sense of [1]. The notion of rigidity in the sense of [1] seems to be considerably stronger than ours. We compare the two in a case when the former is easily manageable. In what follows we use notation and terminology of [1].

Proposition 3.12. Let $\left(W, G_{W}\right)$ be an abstract Witt ring, $-1 \neq-t \in$ $G_{W}$ and $n \geq 2$. If $-t$ is $n$-rigid over $S_{0}$ (relative to $T_{0}=0$ ), then $-t$ is super $n$-rigid (i.e., $x=-t$ satisfies (D)).

Proof. Let $q \in I^{n} W \cap \mathrm{Ann}_{W}\langle 1,-t\rangle$. By [1, Example 1.5.1], $-t$ is $n$-rigid over $S_{0}$ implies $t$ is $n$-rigid over $S_{0}$, hence $I^{n} W=I^{n} S \oplus$ $\langle 1, t\rangle I^{n-1} S$, by [1, Def. 1.4(ii)]. Thus $q=q+\langle 1, t\rangle q_{2}$ with some $q_{1} \in I^{n} S$ and $q_{2} \in I^{n-1} S$. It follows $0=\langle 1,-t\rangle q=\langle 1,-t\rangle q_{1}$. Thus $q_{1} \in I^{n} S \cap$ $\mathrm{Ann}_{W}\langle 1,-t\rangle=0$, by [1, Def. 1.4(iii)], and we get $q=\langle 1, t\rangle q_{2} \in$ $\langle 1, t\rangle I^{n-1} W$. This proves $-t \in \operatorname{Sup} R^{n} W$.

Acknowledgment. The author is indebted to the referee for suggesting condensed versions of proofs of 1.23, 2.8, 2.9, 3.7 and 3.9.

\section{REFERENCES}

[1] J. K. Arason, R. Elman and B. Jacob, Graded Witt rings of elementary type, (to appear).

[2] L. Berman, C. Cordes and R. Ware, Quadratic forms, rigid elements and formal power series fields, J. Algebra, 66 (1980), 123-133.

[3] R. Bos, Quadratic forms, orderings and abstract Witt rings, Thesis, Utrecht 1984.

[4] A. B. Carson and M. A. Marshall, Decomposition of Witt rings, Canad. J. Math., 34 (1982), 1276-1302.

[5] R. Elman, Quadratic forms and the u-invariant, III, Proc. of Quadratic Form Conference, 1976 (G. Orzech, ed.) Queen's Papers in Pure and Applied Math. 46, 422-444, Queen's University, Kingston, Ontario.

[6] R. Elman and T. Y. Lam, Quadratic forms over formally real fields and pythagorean fields, Amer. J. Math., 94 (1972), 1155-1194.

, Quadratic forms and the u-invariant, II, Invent. Math., 21 (1973), 125-137.

21-42.

Quadratic forms under algebraic extensions, Math. Annalen, 219 (1976),

[9] R. Elman, T. Y. Lam and A. R. Wadsworth, Amenable fields and Pfister extensions, Proc. of Quadratic Form Conference, 1976 (G. Orzech, ed.) Queen's Papers in Pure and Applied Math. 46, 445-492, Queen's University, Kingston, Ontario.

[10] _ Function fields of Pfister forms, Invent Math., 51 (1979), 51-75.

[11] _ Pfister ideals in Witt rings, Math. Annalen, 245 (1979), 219-245.

[12] J. Kleinstein and A. Rosenberg, Succinct and representational Witt rings, Pacific J. Math., 86 (1980), 99-137.

[13] T. Y. Lam, The Algebraic Theory of Quadratic Forms, W. A. Benjamin, Reading, Mass., 1973. 
[14] _ Ten lectures on quadratic forms over fields, Proc. of Quadratic Form Conference, 1976 (G. Orzech, ed.) Queen's Papers in Pure and Applied Math. 46, 1-102, Queen's University, Kingston, Ontario.

[15] M. Marshall, Abstract Witt rings, Queen's Papers in Pure and Applied Math. 57, Queen's University, Kingston, Ontario 1980.

[16] O. T. O'Meara, Introduction to Quadratic Forms, Academic Press, New York, 1963.

[17] A. Pfister, Quadratische Formen in beliebigen Körpern, Invent. Math., 1 (1966), 116-132.

[18] R. Ware, Valuation rings and rigid elements in fields, Canad. J. Math., 33 (1981), 1338-1355.

Received May 16, 1985 and in revised form September 19, 1986.

INSTITUTE OF MATHEMATICS

SILESIAN UNIVERSITY

40-007 Katowice, Poland 


\section{PACIFIC JOURNAL OF MATHEMATICS EDITORS}

V. S. VARADARAJAN

(Managing Editor)

University of California

Los Angeles, CA 90024

Herbert Clemens

University of Utah

Salt Lake City, UT 84112

R. FINN

Stanford University

Stanford, CA 94305
ROBION KIRBY

University of California

Berkeley, CA 94720

C. C. MOORE

University of California

Berkeley, CA 94720

HAROLD STARK

University of California, San Diego La Jolla, CA 92093

\section{ASSOCIATE EDITORS}
R. ARENS
E. F. BECKENBACH
B. H. NEUMANN
F. WOLF
K. YosHIDA (1906-1982)

\section{SUPPORTING INSTITUTIONS}

UNIVERSITY OF ARIZONA

UNIVERSITY OF BRITISH COLUMBIA

CALIFORNIA INSTITUTE OF TECHNOLOGY

UNIVERSITY OF CALIFORNIA

MONTANA STATE UNIVERSITY

UNIVERSITY OF NEVADA, RENO

NEW MEXICO STATE UNIVERSITY

OREGON STATE UNIVERSITY
UNIVERSITY OF OREGON UNIVERSITY OF SOUTHERN CALIFORNIA

STANFORD UNIVERSITY

UNIVERSITY OF HAWAII

UNIVERSITY OF TOKYO

UNIVERSITY OF UTAH

WASHINGTON STATE UNIVERSITY

UNIVERSITY OF WASHINGTON 


\section{Pacific Journal of Mathematics}

Vol. 129, No. $1 \quad$ May, 1987

Aldridge Knight Bousfield, Uniqueness of infinite deloopings for

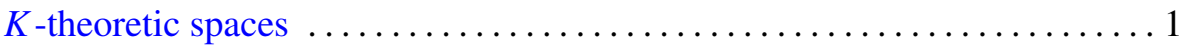

Mark Gregory Davidson, The harmonic representation of $U(p, q)$ and its

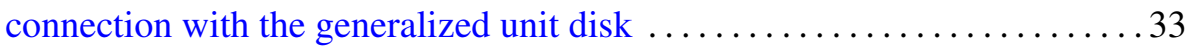

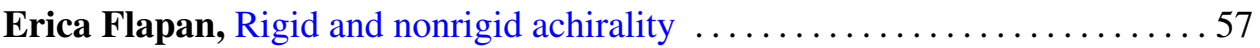

Peter Abraham Greenberg, Pseudogroups of $C^{1}$ piecewise projective

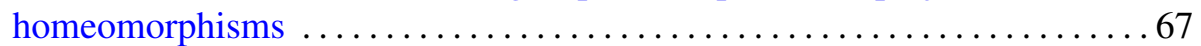

Peter Martin Knopf, Maximal functions on the unit $n$-sphere $\ldots \ldots \ldots \ldots 77$

Norman Jay Levitt and Andrew Ranicki, Intrinsic transversality

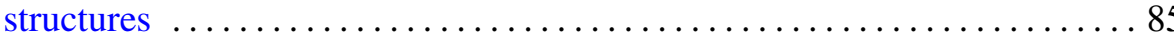

Susan Szczepanski, Invariant submanifolds of free cyclic actions on

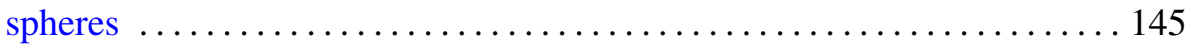

Kazimierz Szymiczek, Generalized rigid elements in fields . . . . . . . . 171

Domingo Toledo, Nonexistence of certain closed complex geodesics in the

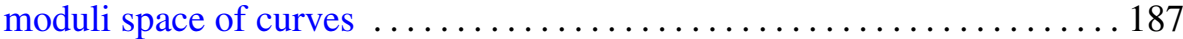

Graham H. Williams, The best modulus of continuity for solutions of the

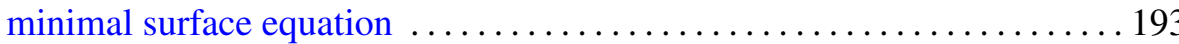

